\title{
Un modelo de desarrollo profesional para la mejora de la reputación corporativa
}

Ana María Casado Molina | acasado1@us.es

UNIVERSIDAD DE SEVILLA

Juan Antonio Peláez Sánchezl jpelaez@coviran.es

COVIRÁN, S. C. A.

Resumen: Actualmente existe una pérdida de confianza en la gestión responsable de las empresas por parte de la sociedad. Dicha percepción es generada por sus públicos externos e internos. Ante esta situación, algunos comités directivos están desarrollando modelos de gestión alternativos dentro de su estrategia de negocio para mejorar la reputación corporativa (RC) percibida por sus empleados. Este trabajo tiene como objetivo la implementación de un modelo de Desarrollo Profesional de $180^{\circ}$ para la mejora de la $R C$ y de las relaciones sostenibles empresa-empleados mediante un Sistema de Desarrollo Profesional (SDP) que promociona el talento de cada empleado e incrementa el valor de la compañía.

Palabras clave: Reputación Corporativa, Gestión de Talento, Estrategia Empresarial.

Abstract: Currently, there is a loss of confidence in the responsible management of companies by society. This perception is generated by its internal and external audiences. In this situation, some management boards are developing alternative management models within their business strategy to improve corporate reputation (CR) with their employees. This work aims to implement a model of $180^{\circ}$ Professional Development to improve the $C R$ and the sustainable relationships between companyemployees through a Professional Development System (PDS), which promotes talent of each employee and contributes to enhance the perception and value of the company.

Key words: Corporate reputation, Talent Management, Business strategy. 


\section{Introducción}

La reputación corporativa (RC) es un valor que se extrae del conjunto de percepciones acerca de la empresa y que tienen los diversos públicos que interactúan con ella, entre los que se pueden distinguir los accionistas, clientes, empleados y otros grupos de interés. Estas percepciones son el resultado del comportamiento desarrollado por la empresa a lo largo del tiempo y describe su capacidad para cubrir las expectativas y aportar valor a los mencionados grupos.

La pérdida de RC impacta directamente en los resultados de las compañías, por lo que las mejoras que se introduzcan en la gestión de su RC redundan directamente en su sostenibilidad y en la del país donde operan.

Por este motivo, son cada vez más las empresas que se preocupan por generar una buena RC con sus públicos, lo que les está llevando a desarrollar políticas que integran en su desempeño la responsabilidad social, la ética empresarial, el buen gobierno corporativo, las relaciones sostenibles y de confianza, la calidad en los procesos, los issues que impactan en el negocio (cuestiones medioambientales, normativas, del mercado, etc.) y modelos de desarrollo profesional entre otros.

Los empleados, uno de los públicos claves de la empresa, aportan valor a la oferta y generan una percepción sobre la compañía. La gestión y desarrollo del talento de este público impacta directamente en una mejor percepción de la profesionalidad, saber hacer y liderazgo de las empresas, y por tanto en una mejora de sus resultados.

En el caso de empresas de servicios, como es el business case que se presenta en este trabajo, los empleados representan el valor que las diferencia en el tiempo y las hace sostenible en un mercado competitivo. En definitiva, los empleados y el buen gobierno de las empresas confeccionan servicios, marcas y empresas con mejor o peor RC.

Son muchas las empresas que están poniendo en práctica estrategias de construcción de su marca como empleadores, con la finalidad de: (1) Atraer a los mejores talentos del mercado de trabajo; y (2) conseguir motivar y retener a los mejores empleados que están en la compañía y que están desarrollando una carrera y trabajo profesional. Esto es lo que se denomina estrategia de Employer Branding (Sánchez y Barriuso, 2007). Para ello, se debe conseguir una armonía entre el sentimiento organizativo interno y la percepción corporativa externa. Se trata de buscar un valor diferencial de la empresa frente a su competencia a través de un posicionamiento reputacional centrado en la atracción, motivación y retención de talento y empleados.

A partir de este análisis situacional, se establece la siguiente hipótesis para este trabajo: La incorporación de los empleados en la estrategia de la empresa a través del desarrollo 
competencias profesionales, consolida la RC interna y contribuye a una mejora de los resultados de productividad.

\section{Objetivos y Metodología}

El objetivo de este trabajo es presentar y analizar un modelo de gestión de la RC interna de las empresas basado en un plan de mejora continua a través de un Sistema de Desarrollo Profesional de $180^{\circ}$.

Para el desarrollo de nuestra investigación, se ofrece en una primera fase una perspectiva de la RC y de los principales monitores que se utilizan actualmente para su gestión, a través del estudio de fuentes bibliográficas secundarias y entrevistas realizadas a empresas de consultoría de RC.

En la segunda fase, se ha desarrollado un modelo propio basado en un Sistema de desarrollo profesional de $180^{\circ}$ para la mejora de la. Este modelo se ha confeccionado a partir de fuentes secundarias y del feedback recibido de expertos en la gestión de equipos en entrevistas individuales, así como de la propia experiencia empresarial de los autores. Este Modelo se ha desarrollado en base a la percepción de dos tipos de públicos internos a través de cuestionarios y entrevistas en profundidad: en primer lugar detectando los intereses de los empleados; y en segundo lugar, las necesidades en competencias profesionales de estas direcciones o áreas de responsabilidad.

En la tercera y última fase, se valida el modelo SDP $180^{\circ}$ con la finalidad de dar respuesta a la hipótesis planteada en esta investigación, mediante la implementación del modelo propuesto en una compañía de distribución de alimentación y la medición de los resultados de productividad obtenidos.

\section{Preliminares}

\subsection{Concepto de $R C$}

La RC puede entenderse como la evaluación global de la organización realizada por sus stakeholders. Esta evaluación es la suma de las percepciones agregadas en relación a la capacidad de la organización para cumplir con sus expectativas (Ferguson et al. 2000; Fombrun, 1996; Groenland, 2002).

Existe un gran número de definiciones sobre el concepto de RC. Para Fombrun y Van Riel (2007: 43) es "la evaluación global de la organización realizada por sus stakeholders. Esta evaluación son percepciones agregadas por los stakeholders en relación a la capacidad de la organización para cumplir con sus expectativas". Según Roberts y Dowling (2002:10771093), la RC es "la percepción de las acciones pasadas y perspectivas futuras de una 
empresa que describen el atractivo global para todos sus participantes cuando se compara con otros rivales". Schultz et al (2001: 24-41) considera la RC corporativa como "el juicio sobre una compañía realizado por un grupo de público a través de sus percepciones y evaluaciones". Para Waddock (2000: 323-345), es "la capacidad percibida del desempeño de una compañía para cumplir con las expectativas de sus públicos". Wartick (2002: 371-392) afirma que la RC es "la agregación de la percepción de cada público individual sobre cómo de buenas son las respuestas de las compañías hacia el cumplimiento de la demanda y las expectativas de muchos de sus públicos estratégicos".

En definitiva, existe un gran número de definiciones diferentes sobre RC aunque la mayoría comparten una serie de conceptos comunes: (1) Son percepciones de los stakeholders; (2) las percepciones son fruto de cómo la empresa satisface las expectativas de sus públicos; y (3) se construye a largo plazo e influye el pasado y el presente de la compañía.

En el ámbito académico, son muchas las investigaciones, y muchos autores, que afirman que la gestión de la RC es un intangible que aporta una ventaja competitiva y provee de muchos beneficios: reducción de costes; incremento de precios y creación de una barrera a la competencia (Dolphin, 2004; Davies et al, 2003); multiplica el valor de las marcas (Black et al, 2000; Fan, 2005), productos y servicios; constituye un escudo frente a la crisis (Villafañe, 2004); transmite compromiso de calidad hacia los consumidores (Fombrun y Shanley, 1990; Bauer et al, 2003) (Berens et al, 2005); facilidad de acceso a nuevos mercados (Fombrun and Riel, 2004); éxito financiero (Fombrun, 2001; De Quevedo et al, 2011; Fuente y Quevedo, 2003); disminuye las amenazas y ataques hacia la marca (Harris Interactive, 2002); disminuye el impacto frente a una crisis; y, promueve la recuperación (Eccles et al, 2007).

Para gestionar la RC las compañías deben trazar políticas de actuación que fomenten relaciones de sostenibilidad con sus principales públicos estratégicos, inversores, clientes, proveedores y empleados. Dentro de esta gestión, se debe trabajar en primer lugar la RC interna o la percepción que los empleados tienen sobre la compañía, para que su comportamiento este alineado con los objetivos de la empresa. Los empleados y su desarrollo profesional, aportan un valor que diferencia a las empresas en el tiempo y en su mercado competitivo. Por eso dentro de las políticas concretas de actuación que ayudan a mejorar la RC interna se deben contemplar modelos de Desarrollo Profesional.

El Desarrollo Profesional, en adelante DP, es un esfuerzo organizado y formalizado que se centra en conseguir tener trabajadores más capacitados para realizar las funciones propias de la empresa. Con la implementación de un modelo de DP los empleados progresan a través de una serie de etapas que se caracterizan por distintas tareas de desarrollo, actividades y relaciones dentro de su empresa. Los modelos de DP benefician tanto a los trabajadores como a la propia empresa. Los empleados descubren sus intereses, habilidades, desarrollan su talento profesional, y aumentan su retribución económica; mientras que la empresa consigue mejorar no sólo sus objetivos económicos sino también su liderazgo y su RC en el 
sector. Las principales prioridades que los empleados tienen en las empresas son (MercoPERSONAS, 2010): salario coherente, desarrollo profesional y promoción interna, motivación y reconocimiento, flexibilidad horaria, buena relación con mandos intermedios, valores éticos y profesionales de la empresa, oportunidad y desarrollo sin discriminación, alta dirección profesional, identificación personal con el proyecto empresarial, y finalmente, empresa con una RC positiva en la sociedad.

\subsection{Monitores de RC}

Para medir la RC las empresas utilizan diferentes herramientas o monitores. Los principales monitores son: MercoPERSONAS (Villafañe, 2010); The Great Place to Work Institute (2011); o modelos específicos adaptados como el Workplace RepTrak WorkRep ${ }^{\text {TM }}$ (Reputation Institute, 2011).

MercoPERSONAS (Villafañe \& Asociados, 2010; Villafañe, 2010) es un monitor que trata de valorar el atractivo de las diferentes empresas como lugares para trabajar, partiendo en este caso de los puntos de vista de diferentes agentes: los trabajadores de las empresas, estudiantes universitarios, antiguos alumnos de escuelas de negocio, la población general y directores de recursos humanos. Asimismo, mercoPERSONAS realiza una comparación de los principales indicadores de gestión de personas, a través de un proceso de benchmarking. Las variables que se consideran para reconocer la RC como Employer Branding sería en base a la rotación externa de la plantilla, la diversidad de sus empleados (nacionalidad, no mujeres, discapacitados, etc.), formación (no horas e inversión en cada empleado), tecnología (porcentaje de la plantilla que usa las TIC), desarrollo profesional (porcentaje de la plantilla en programas de coaching/mentoring), carreras (porcentaje de la plantilla promocionada en el último año), compensación (\% variable sobre la retribución), participación (no de sugerencias/empleado al año) y relaciones laborales (porcentaje de horas perdidas por conflictividad sobre el total de horas trabajas al año).

El monitor The Great Place to Work (2011) analiza el nivel de satisfacción de los empleados en su puesto de trabajo, en base a cuatro variables: (1) Credibilidad en la Estrategia de la empresa; (2) Respeto en relación a todo lo que necesita el empleado para el buen Desarrollo Profesional de su trabajo; (3) Trato Justo, el éxito se reparte equitativamente por medio de programas de compensación y beneficio;(4) Orgullo entre el empleado y la empresa en el entorno de trabajo; (5) y finalmente, Compañerismo, relación entre el empleado y sus compañeros.

Finalmente, el monitor WorkRep (Reputation Institute, 2011) analiza en primer lugar si la empresa por parte de los públicos internos y potenciales empleados es admirada, es estimada, se confía en ella; y en segundo lugar, si sería de interés para trabajar o continuar trabajando en ella. La percepción de los empleados reales o potenciales de una empresa se 
trabajaría en base a cuatro dimensiones que la explican: (1) Condiciones de Trabajo, (2) Actuación y Liderazgo, (3) Productos y RSC, (4) Desarrollo Profesional.

Como se ha mostrado anteriormente, el Desarrollo Profesional es uno de los atributos y/o dimensiones que impactan en los distintos monitores y herramientas de gestión de la RC interna.

\section{Modelo de Desarrollo Profesional}

En este trabajo se propone un modelo de desarrollo profesional de $180^{\circ}$ para la mejora de la RC interna, y de esta manera aportar valor a la empresa. Este Modelo se ha desarrollado en base a dos tipos de públicos internos: en primer lugar los empleados; y en segundo lugar, la dirección o áreas de responsabilidad.

En los empleados se trata de mejorar la percepción que tienen respecto a la empresa. Para ello el modelo propone acciones que trabajen: (1) El talento mediante el desarrollo de nuevas habilidades técnicas y profesionales; (2) Una cultura corporativa implicada en la estrategia corporativa; (3) Un clima laboral favorable y profesional; (4) Una comunicación interna abierta y transparente; y finalmente, (5) la calidad de su trabajo con el desarrollo de nuevas competencias que mejoran los resultados del servicio ofrecido y en definitiva de la empresa.

En la dirección o áreas de responsabilidad, se trata de mejorar la percepción que los empleados tienen acerca de sus responsables inmediatos que contribuyen al logro de la estrategia de la empresa en sus diferentes áreas y a distintos niveles (180\%). Este Modelo de $180^{\circ}$ contempla aspectos de feedback sobre el liderazgo de sus responsables en términos de: (1) Imagen profesional en su comportamiento dentro de la empresa y el cumplimiento de objetivos; (2) Liderazgo en sus competencias de gestión, comunicación e innovación; y finalmente, (3) Dirección participativa en la gestión de equipos.

En la figura 1 se muestra el esquema jerárquico del modelo DP propuesto en este trabajo. Este modelo se caracteriza por:

1) Una estructura jerárquica, que define el valor de la RC interna, a través de sus públicos (Presidencia, Directivos, Jefes de Área y Empleados en general) y de sus respectivas variables.

2) En el caso de Presidencia, Directivos y Jefes de Área se estudian diferentes dimensiones a través de encuestas de percepción a los empleados, midiendo aquellos aspectos que se consideran de interés para el desarrollo de la dirección estratégica de la compañía y la realidad. Además, se contemplan dentro de cada dimensión aquellos atributos que son importantes por la frecuencia de sus comportamientos. Para ello se trabaja bajo un Modelo SDP $180^{\circ}$. 
3) En el caso de los empleados o plantilla en general, se estudian aquellas dimensiones de interés que aportan valor a la empresa y al empleado. En el Modelo SDP se trabaja con entrevistas en profundidad con los empleados donde se conoce los gaps o distancia entre la realidad de los puestos de trabajo y de sus competencias actuales, y las expectativas de sus empleados. Igualmente en cada una de estas dimensiones existen una serie de atributos por los cuales los empleados tienen una percepción favorable o desfavorable hacia su empresa y el comportamiento hacia ellos.

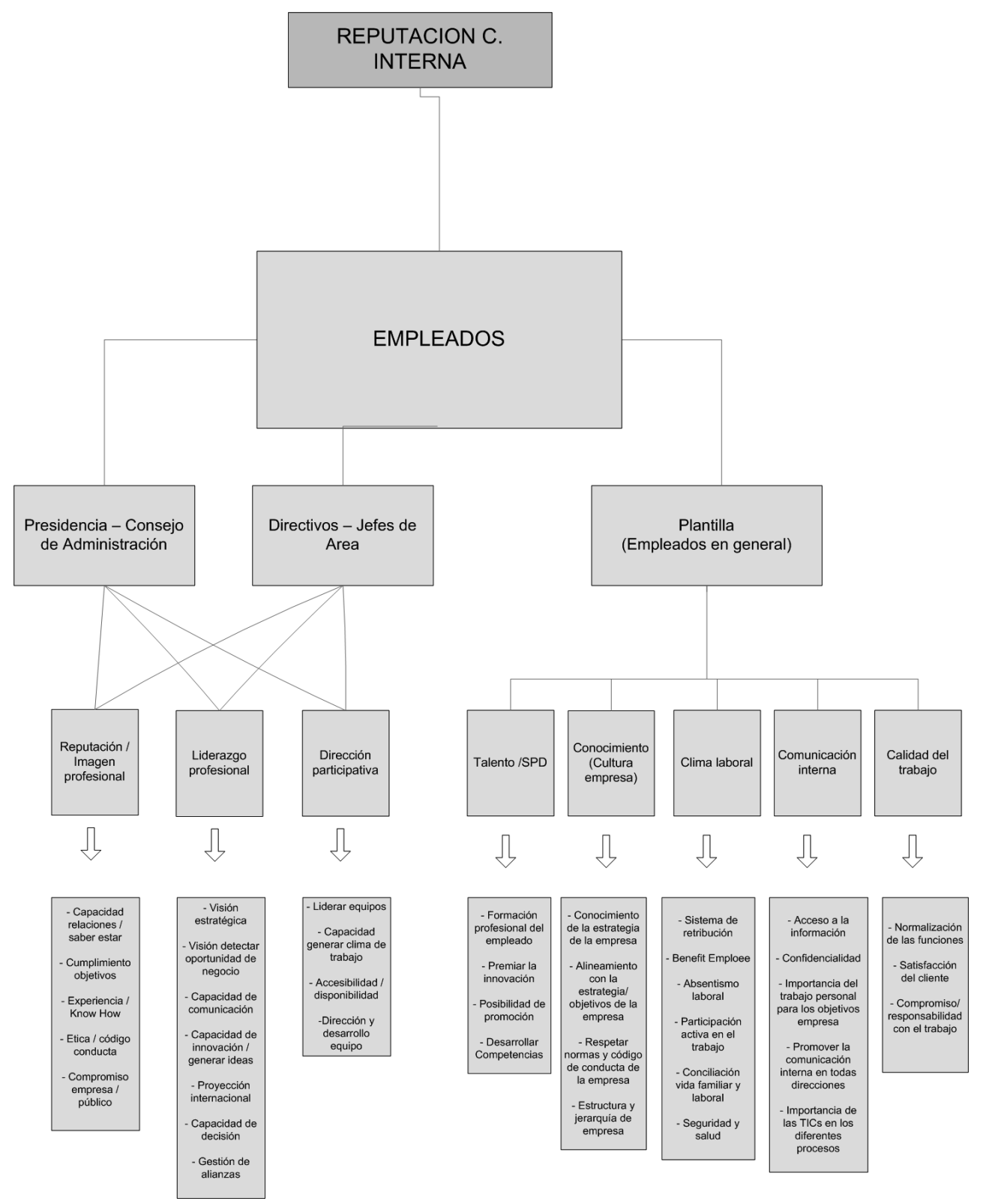

Figura 1. Modelo SDP de RC Corporativa Interna.

Este modelo SDP $180^{\circ}$ de RC interna permite contribuir a la mejora de la RC global mediante: (1) Una marca reconocida y admirada por su capacidad para atraer talento, a través del desarrollo profesional del empleado; (2) Una cultura corporativa coherente y 
participativa con la misión y visión de la empresa; (3) la participación activa interna y el plan de mejora continua de las habilidades de sus empleados, que contribuye a un aumento de productividad; (4) Reducción de costes evitando el nivel de absentismo laboral por estrés o desmotivación; (5) Reducción del índice de rotación de personal; (6) detección de posibles riesgos operacionales, que permiten establecer medidas de control y de ajustes para evitar situaciones de crisis; y finalmente, (7) se generan nuevas oportunidades a través del conocimiento y el desarrollo del talento de los empleados.

\section{Implantación del Modelo}

En este apartado se muestra como llevar a cabo la implantación del modelo DP $180^{\circ}$ de RC. La empresa donde se ha implantado es del sector de la distribución de alimentación con una plantilla superior al millar de empleados.

Los factores que han marcado la implantación son:

1) Diferenciación. La competitividad del sector obliga a buscar un nuevo valor que permita su crecimiento sostenible y su diferenciación en el mercado.

2) Innovación. Una RC basada en los valores de Innovación y Employer Branding.

3) Valor. Las líneas de negocio en las que se enfocan son servicios, donde el empleado aporta valor añadido en su confección. La generación de valor sobre este público tiene un impacto favorable hacia el valor percibido de la empresa por sus clientes.

4) Continuidad. El rápido crecimiento y dispersión geográfica del personal necesita un modelo que fomente la relación continuada "Empresa-Empleado".

\subsection{Fases en la implantación del modelo}

La implantación del modelo, figura 2, consta de una fase inicial llamada Plan de Mejora Continua, y una segunda fase denominada Desarrollo Profesional $180^{\circ}$. Esta segunda fase se implanta una vez consolidado el Plan de Mejora Continua de la primera fase.

En la fase 1, se diseña un Plan de Mejora Continua cuyos objetivos son tratar de mejorar los ratios de productividad de las distintas secciones de la compañía y conseguir un ahorro de costes reduciendo las pérdidas de stock y los consumos energéticos. Los planes de actuación que se ponen en marcha por parte de la empresa para generar una percepción favorable interna por parte de sus empleados y un comportamiento involucrado en el logro de los objetivos son:

Acción 1. Retribución variable por consecución de objetivos del equipo. Esta trata que el empleado mejore su percepción de la empresa, mediante el reconocimiento del esfuerzo 
profesional del equipo, su alineación con la estrategia corporativa y su aportación de valor a través de su experiencia en el desarrollo de posibles medidas de mejora en los procesos.

Acción 2. Cesión de horas de secciones. Esta trata de mejorar los márgenes comerciales de los asociados, mediante la adaptación del empleado a nuevos entornos o situaciones en el trabajo. Con esta acción se disminuye el estrés de los empleados al recibir ayuda de otros compañeros en momentos de saturación de trabajo.

En la Fase 2, se aplica el SDP $180^{\circ}$, y es cuando se consolida una RC interna favorable hacia el comportamiento comprometido de las empresas aportando valor individual al empleado y a todos los niveles de responsabilidad y mandos de la organización. Dentro de este modelo la percepción a generar en el empleado es la aportación de valor individual que la empresa realizaba sobre él. A continuación se detallan los dos grandes objetivos del modelo con sus correspondientes planes de actuación:

Objetivo 1. Fomentar el Talento y las Competencias Individuales en los puestos de trabajo.

Los planes de actuación son:

Acción 1.- Entrevistas periódicas individuales del responsable de área con cada miembro de su equipo para mejorar los procesos de trabajo. Se trata de evaluar las competencias, desempeño y consensuar las mejoras, compromisos y necesidades para conseguirlo. Para ello se pone en marcha un plan de retribución variable que premia la actitud/aptitud individual del empleado por superar las competencias de su puesto de trabajo.

Acción 2.- Plan de formación según necesidad individual para la mejora en el desempeño de su función, y garantizar el crecimiento personal y profesional del empleado.

Objetivo 2. Desarrollar una comunicación transparente y un buen entorno de trabajo.

Los planes de actuación eran:

Acción 1.- Creación de un canal de comunicación entre empleado y nivel superior fluido para fomentar un clima de trabajo positivo y de confianza. A través de éste se conocen aspectos personales $\mathrm{y} / \mathrm{o}$ profesionales que influyen para mejorar sus competencias. Se informa mensualmente de la consecución de sus competencias y se trabajan los recursos individuales necesarios puntuales para ayudarle a conseguirlas.

Acción 2.- Un Sistema de evaluación de los responsables de área para incorporar la participación e integración del personal en la empresa. A través de una valoración 1800, el empleado evalúa a su superior y superiores en función de la frecuencia de comportamientos sobre ciertas competencias. El sistema garantiza la confidencialidad del proceso de recogida de información y se desarrolla de forma impersonal. 


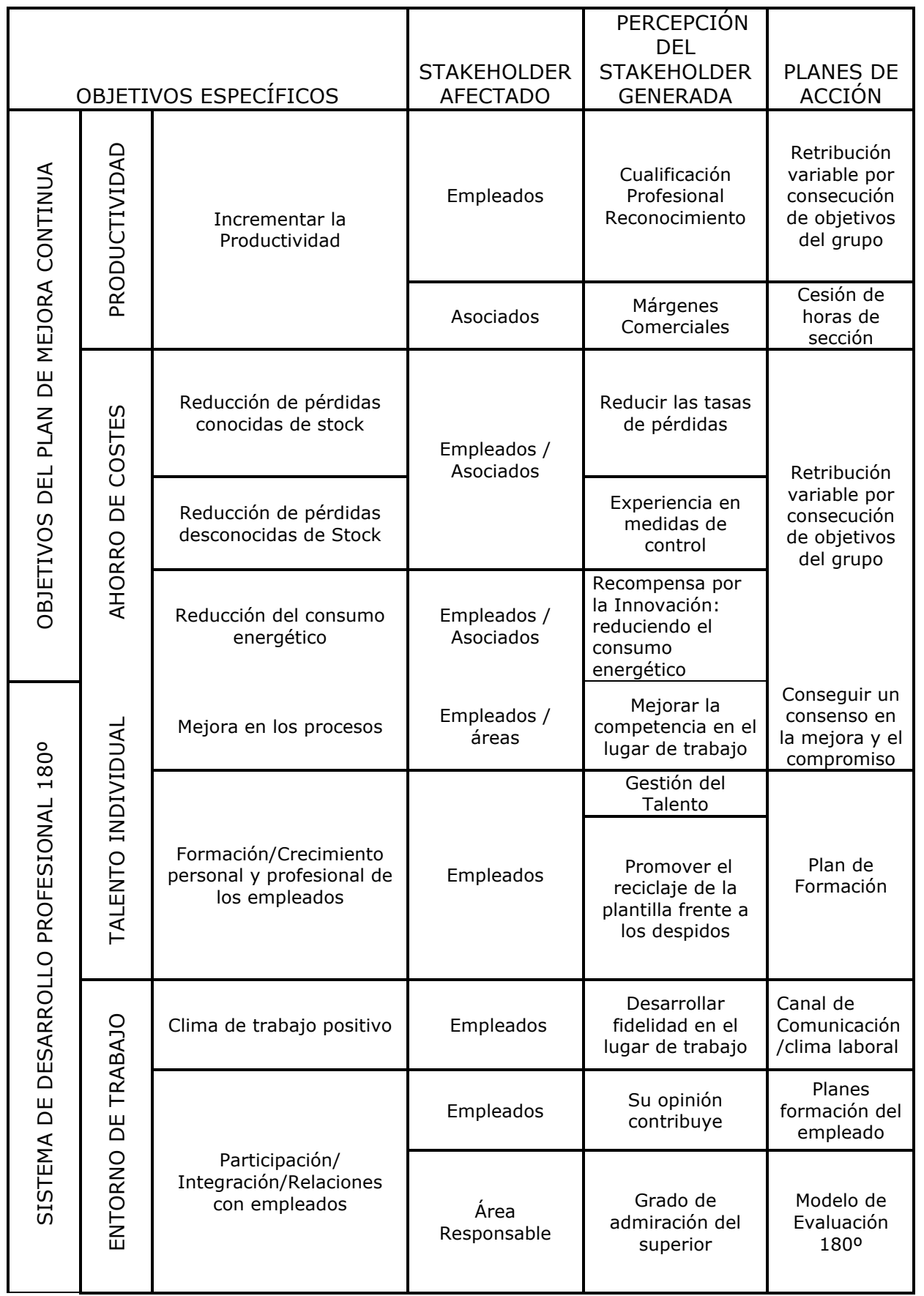

Figura 2. Fases en la implantación del Modelo SDP $180^{\circ}$

La evaluación se hace en base a cinco competencias:

1. Dirección y desarrollo de Personas. Establece con claridad los objetivos del equipo y los logra a través del desarrollo profesional de sus miembros.

2. Orientación al logro. Actitud para realizar bien el trabajo y alcanzar los estándares establecidos. 
3. Comprensión y trato personal. Capacidad para comprender a los colaboradores y dispensarles el trato adecuado.

4. Liderazgo, impacto e influencia. Capacidad de dirigir a los demás hacia una meta común.

5. Comunicación. Capacidad de transmitir la información de forma eficaz.

\subsection{Metodología}

El Modelo trabaja como una oportunidad de que cada trabajador mantenga una conversación con su responsable directo y un seguimiento continuo acerca de cómo desarrolla su trabajo y como ayudarle, además de ser recompensado económicamente por su esfuerzo individual.

La metodología aplicada en el Modelo se caracteriza por trabajar:

1. Entrevistas en profundidad. El Responsable de área la realiza con cada persona de su equipo y tiene una duración de 45 minutos. La periodicidad es semestral y se realiza dos veces al año. El Objetivo es consensuar las competencias a mejorar dentro de su puesto de trabajo y su evaluación periódica para conseguirlas. Previa la realización de la entrevista, se le entrega un manual al entrevistador de cómo realizarla y se le da su formación.

2. Elección de competencias. Para definir todos los tipos de competencia se realizaron entrevistas con todos los directores y responsables, y se definen 11 competencias diferentes para cada puesto de trabajo. Para cada una se establecieron cinco categorías de la $A$ a la $E$, cada opción contiene unos comportamientos que de cumplirse esos trabajadores podrían ser excelentes en el desarrollo de su competencia $(A)$ o deberían mejorar muchísimo $(E)$. Se elaboran 43 cuestionarios distintos en función de las competencias necesarias por puestos de trabajo.

3. Diseño del sistema de puntuación. Después de valorar cada comportamiento, entrevistador y entrevistado deben concertar unas acciones muy concretas que ayuden a mejorar el desempeño de dicha competencia. En la siguiente valoración, se determinará si se han cumplido o no, repercutiendo esto en la puntuación final. Con ello se consigue establecer un sistema que ayude a mejorar de forma directa y continua el desempeño profesional.

4. Diseño del sistema de recompensa económica individual. Para cuantificar esta aportación individual de cada trabajador se seleccionan unas competencias estratégicas, se ponderan y se valora su grado de desempeño.

a. Esa valoración se compara con unos intervalos previamente concebidos. Dichos intervalos trasforman la cuantía inicial que había conseguido el grupo, 
modelándola en un porcentaje de un +/- 30 por ciento. Es decir, si una plataforma de distribución ha conseguido, a través de sus objetivos, una cuantía hipotética para sus trabajadores de $100 €$, el trabajador, dependiendo de su valoración, puede obtener desde $70 €$ hasta $130 €$.

b. El porcentaje obtenido está supeditado a la consecución de los objetivos grupales. Si no se cumplen los objetivos grupales, ningún miembro del equipo recibirá retribución, independientemente de la puntuación obtenida en la valoración.

5. Tratamiento del feedback. En esta entrevista se obtiene mucha información sobre el personal, específicamente en el apartado de observaciones y además se detectan las necesidades formativas individuales para un mejor desarrollo de sus competencias en el puesto de trabajo.

6. Valoración $180^{\circ}$. Los empleados realizan la evaluación de mandos de la organización, mediante un cuestionario. Además no se realiza bajo entrevista directa, el cuestionario no es identificable, se entrega en sobre cerrado y se recoge de la misma forma o se rellena en el portal del empleado. Se preserva la privacidad del empleado que evalúa. La escala de valoración se definió bajo tres intervalos de calidad del desempeño (frecuencia del comportamiento): De 8 a 10, desempeño excelente; De 7 a 8: desempeño satisfactorio; De 0 a 7: un desempeño insatisfactorio.

\section{Resultados del Modelo SDP}

Los resultados obtenidos después de la implantación del Modelo SDP $180^{\circ}$ son los siguientes:

\section{Resultados Cualitativos.}

Como muestra la figura 3, de los objetivos definidos en la fase 2 del Modelo SDP $180^{\circ}$ se desprenden unos resultados que impactan directamente sobre la RC interna de la compañía, porque mejoran la percepción que los públicos internos tienen sobre la empresa o workplace.

\begin{tabular}{|c|c|}
\hline $\begin{array}{c}\text { Objetivo } 1 . \\
\text { Promover el Talento y } \\
\text { la competencia } \\
\text { individual en el puesto } \\
\text { de trabajo }\end{array}$ & $\begin{array}{l}\text { Resultados } \\
\text { (Percepciones favorables del Empleado) } \\
\text { 1. La compañía se preocupa por mejorar la } \\
\text { competencia en el puesto de trabajo } \\
\text { 2. Recompensa el esfuerzo y la contribución personal } \\
\text { 3. Promueve el reciclaje y no el despido por } \\
\text { obsolescencia del empleado } \\
\text { 4. Apuesta por el desarrollo profesional del empleado }\end{array}$ \\
\hline $\begin{array}{c}\text { Objetivo } 2 . \\
\text { Desarrollar canales de } \\
\text { comunicación } \\
\text { transparentes y un buen } \\
\text { entorno de trabajo. }\end{array}$ & $\begin{array}{l}\text { Resultados } \\
\text { (Percepciones favorables del empleado) } \\
\text { 1. Confianza en la compañía donde trabaja } \\
\text { 2. Su opinión se tiene en cuenta y contribuye a la } \\
\text { Innovación } \\
\text { 3. Añade valor al negocio de la compañía } \\
\text { 4. Admira el desempeño y el liderazgo profesional de } \\
\text { su superior inmediato (Director, Responsable, etc.). }\end{array}$ \\
\hline
\end{tabular}


Figura 3. Resultados Cualitativos del Modelo SDP $180^{\circ}$

\section{Resultados Cuantitativos.}

Estas percepciones favorables del empleado o RC interna favorable, generadas a través del modelo SDP $180^{\circ}$, se traducen en comportamientos implicados del personal hacia el logro de los objetivos de la empresa en el tiempo. Por lo tanto el desarrollo de un modelo SDP $180^{\circ}$ de RC interna atrae ingresos y reduce costes en las compañías.

En relación a los resultados sobre los objetivos de la empresa, descritos en la fase 1 del Plan de Mejora Continua fueron los siguientes:

a) Evolución en la mejora de la productividad avalan el Modelo SDP:

Durante los dos primeros años de implantación en términos globales (medidos en unidades de producción) la productividad mejoró un 5,34\%. Esta mejora se acentuó aún más en algunas plataformas no nodrizas con crecimientos del 22,41\% o del 9,52 \%. El crecimiento en alguna plataforma nodriza llegó en conjunto al 7,44\%, siendo en alguna de sus secciones un crecimiento tan espectacular como el $21,76 \%$.

La productividad de los empleados en estos dos años ha experimentado un crecimiento del 10’26\% (medidas en unidades de producción).

b) Descenso en las pérdidas de inventario. La reducción global de la pérdida de inventario en estos años fue del $20,75 \%$. Esta reducción alcanzó en algunas de las plataformas no nodrizas unas cifras medias del 44,24\%.

\begin{tabular}{|c|c|c|c|c|}
\hline Resultados & Año 1 & Año 2 & Año 3 & Año 4 \\
\hline $\begin{array}{l}\text { Número de Empleados con Retribuc. } \\
\text { Variable sobre el total y por áreas }\end{array}$ & $15 \%$ & $50 \%$ & $80 \%$ & $98 \%$ \\
\hline $\begin{array}{l}\text { \% incremento cesión de horas entre } \\
\text { secciones, evitando nuevas } \\
\text { contrataciones y reduciendo el nivel de } \\
\text { estrés del empleado }\end{array}$ & $\mathrm{n} / \mathrm{s}$ & $10,15 \%$ & $16,60 \%$ & $19,72 \%$ \\
\hline $\begin{array}{l}\text { \% de Reducción Perdidas Conocidas } \\
\text { (ratio) }\end{array}$ & $2 \%$ & $10 \%$ & $9 \%$ & $5 \%$ \\
\hline $\begin{array}{l}\text { \% de Reducción Perdidas Desconocidas } \\
\text { (ratio) }\end{array}$ & $4 \%$ & $5 \%$ & $4,5 \%$ & $2 \%$ \\
\hline $\begin{array}{l}\text { \% de Ahorro Consumo Energético } \\
\text { (ratio) }\end{array}$ & $\mathrm{n} / \mathrm{s}$ & $\mathrm{n} / \mathrm{s}$ & $2,15 \%$ & $1,25 \%$ \\
\hline Número de cursos por personal y áreas & \multicolumn{4}{|c|}{$\begin{array}{c}1 \text { curso por persona y año en competencias } \\
\text { concretas }\end{array}$} \\
\hline $\begin{array}{l}\text { Número Empleados Reciben } \\
\text { Recompensa Indiv. sobre el total y por } \\
\text { áreas }\end{array}$ & $\begin{array}{l}\sin \\
\text { datos. }\end{array}$ & sin datos & $33,27 \%$ & $38,91 \%$ \\
\hline
\end{tabular}

Tabla 1. Resultados Cuantitativos del Modelo SDP $180^{\circ}$ (2007-2010)

La tabla 1 muestra los diferentes resultados obtenidos desde el primer año de implantación del modelo. Estos datos muestran que a partir del tercer año, una vez implantada la segunda fase del modelo, los ratios de retribuciones variables aumentan, se incrementan también los 
ratios de recompensa individual y la cesión de horas entre secciones optimizando la productividad en las diferentes áreas y evitando el estrés del empleado.

Respecto a la reducción de pérdidas y ahorro energético, se puede observar el ahorro obtenido. Además se observa que fruto de la gestión de un modelo SPD $180^{\circ}$, la implicación de la plantilla de empleados se refleja en la tendencia a incrementarse los porcentajes de empleados que consiguen la retribución variable del equipo y su recompensa individual.

En cuanto al impacto del modelo SDP $180^{\circ}$ en el reconocimiento económico del personal observamos en la figura 4 los siguientes resultados:

1. Un $9,48 \%$ de los trabajadores cobraron el $130 \%$ (el $100 \%$ del variable por consecución de objetivos del grupo y un $+30 \%$ por desarrollo individual)

2. Un $23,59 \%$ cobraron el $110 \%$ ( $+10 \%$ por desarrollo individual $)$

3. Un $56,25 \%$ cobraron el $100 \%$ ( no fueron premiados por desarrollo individual)

4. Un $9,48 \%$ cobraron el $80 \%$ (Se restó un $20 \%$ del variable del grupo por no alcanzar las competencias individuales)

5. Un $1,01 \%$ cobraron el $70 \%$ (En este caso, estos trabajadores no cumplieron con las competencias individuales y se aplicó sobre el variable del equipo -30\%)

Finalmente, el modelo consigue una tasa de rotación de personal por debajo del 0,05\%. Esto refleja la relación sostenible entre Empresa-Trabajador que se genera a través de la implementación del modelo SPD $180^{\circ}$.

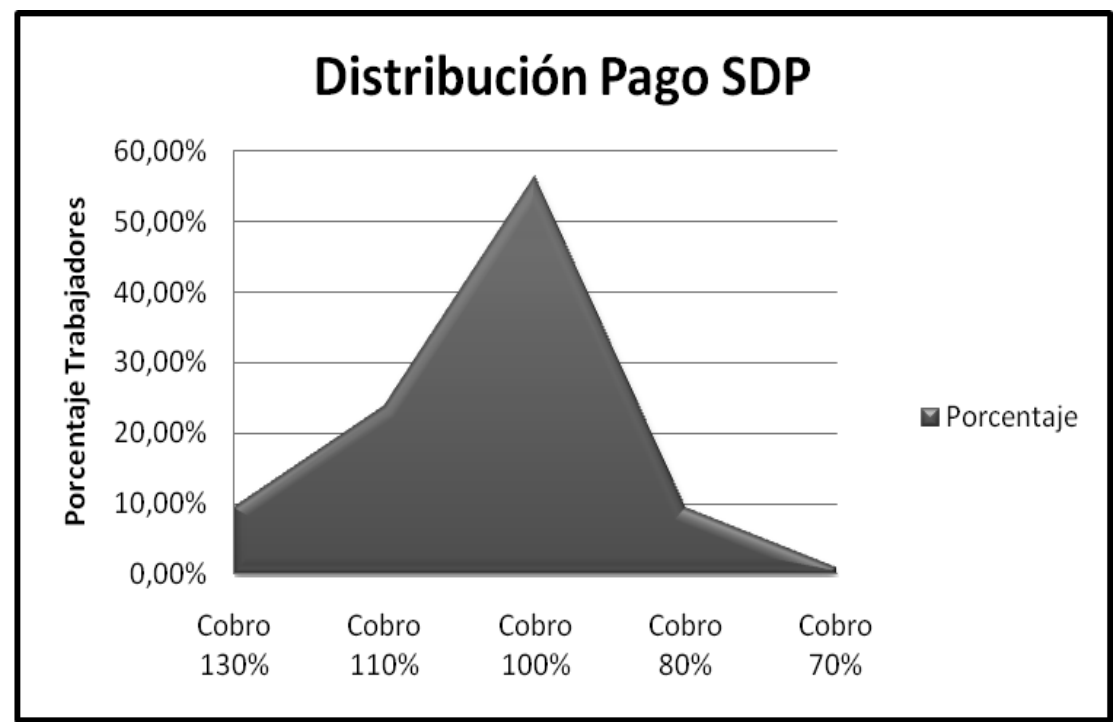

Figura 4. Impacto del Modelo SDP en el Reconocimiento Económico Individual 
Todos estos resultados, cualitativos y cuantitativos, muestran que la implantación de este tipo de modelos SDP $180^{\circ}$ genera una RC interna y contribuye a mejorar los resultados económicos.

\section{Conclusiones}

En este trabajo se ha presentado un modelo de Desarrollo Profesional de $180^{\circ}$ para la mejora de la RC Interna y las relaciones sostenibles Empresa-Empleados. El Modelo desarrolla aportaciones de valor que beneficien tanto al empleado como a la empresa, generando una percepción interna favorable y proactiva que contribuye a la mejora continua de sus objetivos y de su RC.

El modelo consiste en un sistema de desarrollo continuo de las competencias profesionales individuales de cada empleado y específicas para cada puesto de trabajo para mejorar los objetivos de la compañía. Este Modelo consta de dos fases: La primera está orientada hacia un Plan de Mejora de los ratios de productividad y una reducción de costes logísticos, a través de un sistema de retribución variable para los empleados por equipo de trabajo. La segunda fase está orientada al Desarrollo Profesional Individual mediante: la implantación de un canal de comunicación entre responsables y empleados que promueve el desarrollo de las competencias individuales; un plan de formación específico para mejorar su cualificación y habilidades personales; un sistema de retribución individual por logro de competencias personales; y finalmente, un sistema de evaluación de las competencias profesionales de los responsables o directores de área.

El modelo ha sido implantado en una compañía de distribución de alimentación, con una plantilla que supera los 1000 empleados. La implementación de este modelo ha mostrado los siguientes resultados: (1) La ejecución de un gran número de cursos ad-hoc y la recompensa económica individual a más de un 30\% de su plantilla por su esfuerzo profesional, genera una percepción interna de la empresa como marca comprometida con su personal; (2) La productividad aumenta de manera significativa; (3) Se produce una reducción de costes con un ahorro de consumo energético y un descenso considerable de las pérdidas de inventario; (4) La rotación del personal disminuye de manera significativa, fidelizando al empleado con la empresa, generando de esta manera relaciones sostenibles en el tiempo y consolidando su cultura y su identidad corporativa; (5) Se disminuyen costes de personal al incrementarse la cesión de horas de empleados entre secciones, y se reduce el absentismo laboral provocado por el estrés en los empleados; y finalmente, (6) la mejora y evaluación continua del desarrollo profesional individual a todos los niveles -empleados y responsables- permite a la empresa estar preparada para afrontar nuevos retos.

Los resultados alcanzados confirman que la implementación del modelo en los empleados de la empresa, mejora su RC interna como Employer Branding, y sus resultados de productividad. 


\section{REFERENCIAS BIBLIOGRÁFICAS}

BAUER, Hans H., HAMMERSCHMIDT, Maik y BRAEHLER, Matthias (2003): "The Customer lifetime value concept and its contribution to corporate valuation", en Yearbook of Marketing and Consumer Research, no 1, pp. 47-67.

BERENS, Guido, RIEL, Cees B.M. van and BRUGGEN, Gerrit H. van (2005): "Corporate associations and consumer product responses: the moderating role of corporate brand dominance", en Journal of Marketing, no 69, pp. 3548.

BLACK, Ervin L., CARNES, Thomas A. and RICHARDSON, Vernon H. (2000): "The marketing valuation of corporate reputation", en Corporate Reputation Review, vol. 31, no 1, pp. 31-41.

DAVIES, Gary, CHUN, Rosa, SILVA DA, Rui Vinhas and Roper, Stuart (2003): Corporate Reputation and Competitiveness, London, Routledge.

DE QUEVEDO, Esther, DELGADO, Juan B. y DE LA FUENTE, Juan M. (2011): "Financial Impacts of Corporate Reputation", en Helm S. et al. (Eds.), Reputation Management, Management for Professional, New York, Springer, pp. 163-178.

DOLPHIN, Richard R. (2004): "Corporate Reputation - a Value Creating Strategy", en Corporate Governance, no 4, pp. 77-92.

ECCLES, Robert G., NEWQUIST, Scott C. y SCHARTZ, Roland (2007): "Reputation and its Risks", en Harvard Business Review, vol. 85, no 2, pp. 104-114

FAN, Ying (2005): "Ethical branding and corporate reputation", en Corporate Communications: An International Journal, vol. 10, no 4, pp. 341-350.

FERGUSON, Tyler David, DEEPHOUSE, David L. y FERGUSON, William L. (1972): "Do strategic groups differ in reputation?", en Strategic Management Journal, vol 21, no 12, pp. 1195-1214.

FOMBRUN, Charles J. (2001): "Corporate Reputation as economic assets", en Freeman,R.E. y Harrison, J.S. (eds), The Blackwell Handbook of Strategic Management, USA, Blackwell, p. 293.

FOMBRUN, Charles J. (1996): Reputation: Realizing value from the corporate image, Boston, Harvard Business School Press.

FOMBRUN, Charles J. and Riel, Cees B.M. van (2004): "Fame \& Fortune. How successful companies build winning reputations", New York, Pearson Education.

FOMBRUN, Charles J. y SHANLEY, Mark (1990): "What's in a Name? Reputation Building and Corporate Strategy", en Academy of Management Journal, no 33 , pp. 233-258. 
FUENTE, Juan M. Y DE QUEVEDO, Esther. (2003): "Empirical Analysis of the Relationship between Corporate Reputation and Financial Performance: A Survey of the Literature", en Corporate Reputation Review, n 6, pp. 161177.

GROENLAND, Edward. A.G. (2002): "Qualitative, research to validate the RQDimensions", en Corporate Reputation Review, vol. 4, no 4, pp. 209-315.

HARRIS INTERACTIVE (2002): Fourth Annual Corporate Reputation Report: CEOs on Corporate Reputation Influencers, Corporate Social Responsibility and Borard-Level Involvement, USA, Harris Interactive and Hill \& Knowlton.

REPUTATION INSTITUTE (2011). Estudio Reptrak Pulse(Reptrak Press, 2011), en http://www.reputationinstitute.com/advisory-services/workrep (consultado 22/09/2012)

RIEL, Cees B.M. van y FOMBRUN, Charles J. (2007): Essentials of Corporate Communication. Implementing practices for effective reputation management, New York, Routledge.

ROBERTS, Peter W. y DOWLING, Grahame R. (2002): "Corporate Reputation and Sustained Superior Financial Performance", en Strategic Management Journal, vol. 23, no 12, pp. 1077-1093.

SÁNCHEZ, Ma Isabel, BARRIUSO, Ma Cristina, (2007): "Explorando la relación entre la RC Corporativa y el Employer Branding", en Ayala, J.C., Conocimiento, innovación y emprendedores : camino al futuro, La Rioja, Universidad de La Rioja, pp. 3144-3154.

SHULTZ, Majken, MOURITZEN, Jan. y GABRIELSEN, Gorm (2001): "Sticky Reputation: Analyzing a ranking system", en Corporate Reputation Review, vol. 4, no 1, pp. 24-41.

THE GREAT PLACE TO WORK INSTITUTE (2011). Las mejores empresas para trabajar en España (Ranking), Marzo 22, 2011 en http://www.greatplacetowork.es/great/dimensiones.php (consultado: 22/11/2012)

VILLAFAÑE, Justo (2010): Informe Anual 2010. La comunicación empresarial y la gestión de los intangibles en España y Latinoamérica, Madrid, Editorial Pearson.

VILLAFAÑE \& ASOCIADOS (2010): Las Mejores Empresas para Trabajar en España - Ranking, Octubre 20, 2010 en http://www.villafane.info/files/ Presentacion_Merco_Personas_2010.pdf (consultado 20/02/2011)

VILLAFAÑE, Justo (2004): La Buena RC. Claves del valor intangible de las empresas, Madrid, Pirámide. 
WADDOCK, Sandra (2000): "The multiple bottom lines of corporate citizenship: Social investing, reputation, and responsibility audits", en Business and Society Review, no 105, pp. 323-345.

WARTICK, Steven L. (2002) 'Measuring Corporate Reputation', en Business \& Strategy, no 41, pp. 371-392.

[Recibido: 7 de mayo de 2013. Aceptado: 7 de septiembre de 2013]. 